Canadian Oncology

Nursing Journal

Revue canadienne

de soins infirmiers

en oncologie

Volume 29, Issue 1 - Winter 2019

elSSN: 2368-8076 


\title{
Étude en population de la fréquence de navigation et de contact pour soins palliatifs avant le décès de patients atteints de cancer
}

\author{
par Grace M. Johnston, Grace Park, Robin Urquhart, Gordon Walsh, Meg McCallum et Krista Rigby
}

\section{RÉSUMÉ}

Au Canada, la navigation des patients atteints de cancer a vu le jour en Nouvelle-Écosse, en 2002, à l'instigation d'infirmières en oncologie ayant décidé de soutenir les patients du diagnostic au décès. La présente étude voulait déterminer la fréquence (et la variation de fréquence) des services de navigation et de contacts pour soins palliatifs chez les adultes décédés entre 2011 et 2014 après avoir reçu un diagnostic de cancer ainsi que des services de navigation. Parmi les 2532 sujets de l'étude, 56,7\% ont bénéficié de services de navigation pendant plus d'un mois et 30,6\% ont eu un contact pour soins palliatifs. Certaines variations ont été observées entre les régions géographiques, les stades de cancer, le temps écoulé entre le diagnostic et le décès, et la cause du décès (attribuable ou non au cancer). Il serait bon d'étudier plus en détail le rôle de la navigation pour les personnes en fin de vie.

\section{AU SUJET DES AUTEURS}

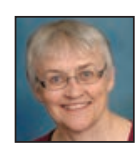
Grace M. Johnston, Ph.D., Enregistrement et analyses de données sur le cancer, Programme de soins du cancer de la Nouvelle-Écosse, Régie de la santé de la Nouvelle-Écosse et École d'administration de la santé, Faculté de la santé, Université Dalhousie, Halifax, Nouvelle-Écosse

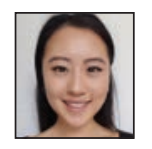

Grace Park, B.Sc.inf., inf. aut., MD(c), Faculté de médecine, Université Dalhousie, Halifax, Nouvelle-Écosse

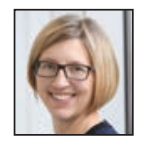

Robin Urquhart, Ph.D., Faculté de médecine, Université Dalhousie, Halifax, Nouvelle-Écosse

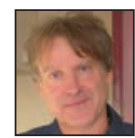

Gordon Walsh, M.Sc., Programme de soins du cancer de la Nouvelle-Écosse, Régie de la santé de la Nouvelle-Écosse, Halifax, Nouvelle-Écosse

Meg McCallum, M.A., Programme de soins du cancer de la Nouvelle-Écosse, Régie de la santé de la Nouvelle-Écosse, Halifax, Nouvelle-Écosse

Krista Rigby, B.Sc.inf., inf. aut., M.G.S.S., Programme de soins du cancer de la Nouvelle-Écosse, Régie de la santé de la NouvelleÉcosse, Halifax, Nouvelle-Écosse

Auteure-ressource: Grace M. Johnston, Ph.D., professeure émérite, École d'administration de la santé, Faculté de la santé, édifice Sir Charles Tupper Medical Building, 2e étage, 5850 College Street, C. P. 15000, Halifax (Nouvelle-Écosse) B3H 4R2 Tél. : 902-835-9387

Grace.Johnston@dal.ca

DOI: $10.5737 / 236880762912533$

\section{INTRODUCTION}

F nviron $50 \%$ des Canadiens recevront un diagnostic de cancer au cours de leur vie et quelque $25 \%$ seront emportés par cette maladie (Société canadienne du cancer [SCC], 2017). Selon Fitch (2017), le nombre de décès attribuables au cancer augmentera au cours des prochaines années en raison de notre population vieillissante. Les personnes en fin de vie auront sans doute besoin d'aide pour naviguer le système de santé. Par conséquent, la présente étude cherchait à déterminer la fréquence (et ses variations) de recours aux services de navigation et de contact pour soins palliatifs chez les patients décédés des suites du cancer.

Les infirmières pivots prodiguent aux patients atteints de cancer et à leur famille de l'enseignement, de l'aide pour contrôler les symptômes, du soutien pratique et financier, de même que des services de coordination des soins tout au long de la trajectoire du cancer, du diagnostic jusqu'aux aux soins palliatifs et terminaux (Fillion et al., 2012). Au départ, le rôle de l'infirmière pivot était surtout d'accélérer le dépistage et le diagnostic du cancer chez les populations vulnérables (Paskett, Harrop et Wells, 2011; Freeman et Rodriguez, 2011), mais la navigation est maintenant préconisée pour faciliter le recours aux soins palliatifs et la planification préalable des soins pour les personnes atteintes de cancer ou d'autres maladies évolutives limitant leur espérance de vie (Hauser et al., 2011).

La Nouvelle-Écosse, le Québec (Fillion et al., 2012) et l'Alberta (Watson, Vimy, Anderson, Champ et DeIure, 2016) ont publié une description de leurs programmes de navigation, mais les statistiques sur les populations d'usagers des services de navigation, elles, demeurent rares. Certaines études en population ont évalué la participation à des programmes de soins palliatifs des personnes mourantes atteintes de cancer (Gao, Johnston, Lavergne et McIntyre, 2011; Craigs, West, Hurlow, Bennett et Ziegler, 2018). Toutefois, la présente étude et le document complémentaire (Park, Johnston, Urquhart, Walsh et McCallum, 2018) sont les premiers à employer ce type d'approche pour examiner l'apport aux soins palliatifs de l'infirmière pivot en oncologie.

Pour que toutes les personnes qui en ont besoin reçoivent des soins palliatifs de bonne qualité, il est essentiel d'analyser les populations concernées (SCC, 2010, p. 70 et 78). La présente étude a pour objectifs de connaître la fréquence de navigation et de contact pour soins palliatifs des patients adultes de Nouvelle-Écosse atteints de cancer ayant reçu des services de navigation et succombé à la maladie. Létude tente également d'établir la cause des variations de fréquence en fonction des facteurs démographiques, des caractéristiques pathologiques 
et de l'issue de la maladie. Comme nous savons que les taux d'accès des patients cancéreux à d'autres formes de soins palliatifs sont influencés par ces mêmes facteurs, caractéristiques et issues (Lavergne, Johnston, Gao, Dummer et Rheaume, 2011), nous avons supposé que la fréquence de navigation et de contact pour soins palliatifs pouvait elle aussi varier pour les mêmes raisons.

\section{MÉTHODOLOGIE}

\section{Contexte de l'étude}

Au Canada, c'est en Nouvelle-Écosse, en 2002, que des infirmières d'expérience en oncologie ont commencé à offrir des services de navigation (Walkinshaw, 2011; Fillion et al., 2012). Nous disposons donc de données longitudinales sur les personnes en fin de vie. Récemment, nous avons comparé les patients atteints de cancer ayant reçu des services de navigation à ceux qui n'en avaient pas bénéficié (Park, Johnston, Urquhart, Walsh et McCallum, 2018). La présente étude utilise ces mêmes données, en ne retenant que les patients ayant obtenu des services de navigation, le but étant de déterminer la fréquence de navigation et de contact pour soins palliatifs.

De multiples interactions entre les infirmières pivots et les patients peuvent avoir lieu sur une courte période de temps. Pour réduire au minimum le temps consacré à la gestion des dossiers, les infirmières pivots présentent au programme provincial de soins du cancer un seul compte rendu par mois pour chaque patient ayant reçu des services de navigation; l'information est ensuite codée dans le Système d'enregistrement de données sur le cancer. La présente étude cherchait à savoir si les infirmières pivots avaient été en contact avec les patients atteints de cancer (ou avec quelqu'un d'autre au sujet de ces patients) pendant un temps relativement court ou sur une plus longue période.

Les faits constatés et rapportés dans les registres de navigation mensuels sont regroupés en catégories, notamment « Coordination » et « Contacts/aiguillage ». Sous ces deux catégories figure une rubrique intitulée « Soins palliatifs », qui se trouve aussi sous la catégorie « Personne ayant fait la demande d'aiguillage ». Aux fins de la présente étude, un « contact pour soins palliatifs » était codé chaque fois que l'une de ces catégories était cochée dans le dernier dossier de navigation d'un patient. Comme il y a des équipes spécialisées en soins palliatifs partout en province, il est présumé qu'un aiguillage relatif aux soins palliatifs concerne l'équipe de soins palliatifs d'une région donnée.

\section{Devis de l'étude et collecte de données}

Une étude quantitative en population a été montée à partir de données administratives déjà collectées. Leétude adoptait un devis rétrospectif et, à partir du décès des patients, remontait leur parcours pendant toute leur expérience du cancer afin de trouver les renseignements nécessaires sur la navigation, les facteurs démographiques, les caractéristiques pathologiques et l'issue de la maladie. Toutes les données provenaient du Système d'enregistrement de données sur le cancer de la Régie de la santé de la Nouvelle-Écosse. Ce registre recense tous les nouveaux cas de cancers invasifs diagnostiqués dans la province depuis le début des années 1970, l'historique de navigation, ainsi que l'information figurant sur le certificat de décès.

\section{Population à l'étude}

Étaient inclus dans la population à l'étude tous les adultes (19 ans et plus) néo-écossais qui avaient reçu un diagnostic de cancer invasif et des services de navigation entre 2002 et 2014, et qui étaient par la suite décédés entre 2011 et 2014. Tous les patients ayant reçu un diagnostic de cancer ont été intégrés à l'étude, que le décès soit attribuable ou non au cancer. Toutefois, les cas constitués uniquement d'un certificat de décès ont été écartés, le diagnostic ayant été posé à la date du décès. Nous n'avons pas retenu non plus les données concernant le centre de la province, car les patients atteints de cancer n'ont pas tous accès à des services de navigation dans cette région. Ont également été exclus les patients de l'est de la Nouvelle-Écosse décédés en 2011-2012, la mise en œuvre complète des services de navigation n'y remontant qu'à 2011.

\section{Variables}

Aux fins de la présente étude, la fréquence de navigation était dichotomisée selon que les dossiers mensuels indiquaient « une » ou «plusieurs » occurrences de navigation. Les variables démographiques (âge au moment du décès, sexe, région géographique de résidence et année du décès) ont été obtenues à partir des certificats de décès de l'état civil provincial. Trois variables propres à la maladie (diagnostic de cancer le plus rapproché du décès, stade au moment du diagnostic et diagnostic antérieur de cancer) ont été extraites du système provincial d'enregistrement de données sur le cancer. Le certificat de décès permettait également de savoir si le patient était mort à cause du cancer. La différence entre la date du dernier diagnostic de cancer figurant dans le système d'enregistrement de données sur le cancer et la date inscrite sur le certificat de décès donnait quant à elle le temps écoulé entre le diagnostic et le décès. La dernière variable, qui concerne l'issue de la maladie (décès à l'hôpital ou non), provient elle aussi du certificat de décès.

\section{Analyse des données}

Les comptes de fréquence et la proportion de patients répondant à chaque caractéristique ont été saisis dans Excel. Les statistiques ont ensuite été vérifiées à l'aide du programme SAS. Pour protéger la confidentialité des sujets de l'étude, les catégories comptant moins de cinq résultats n'ont pas été rapportées. Des tests de khi carré ont été réalisés à l'aide du programme SAS. Une valeur p inférieure à .05 indiquait des variations de taux statistiquement significatives. Létude a été approuvée par le comité d'éthique de la recherche de la Régie de la santé de la Nouvelle-Écosse.

\section{RÉSULTATS}

Parmi les 2532 patients décédés de l'étude, 1436 (56,7 \%) avaient plus d'un dossier de navigation (tableau 1). Pour 776 des patients (30,6\%), le dernier dossier de navigation mensuel mentionnait un contact pour soins palliatifs (tableau 2). Dans près de la moitié $(47,9 \%)$ des contacts pour soins palliatifs, 


\begin{tabular}{|c|c|c|c|}
\hline \multirow[t]{2}{*}{ Caractéristiques des patients (décédés) } & \multicolumn{2}{|c|}{ Plus d'un dossier de navigation } & \multirow[t]{2}{*}{ Total } \\
\hline & Nombre & $\%$ & \\
\hline \multicolumn{4}{|c|}{ CARACTÉRISTIQUES DÉMOGRAPHIQUES } \\
\hline \multicolumn{4}{|l|}{ Tranche d'âge au moment du décès } \\
\hline 19-39 & 13 & 72,2 & 18 \\
\hline $40-54$ & 155 & 66,0 & 235 \\
\hline $55-69$ & 612 & 64,6 & 947 \\
\hline $70-79$ & 426 & 54,1 & 787 \\
\hline $80-89$ & 190 & 42,7 & 445 \\
\hline $90+$ & 39 & 39,0 & 100 \\
\hline \multicolumn{4}{|l|}{ Sexe } \\
\hline Femme & 699 & 57,3 & 1220 \\
\hline Homme & 736 & 56,1 & 1312 \\
\hline \multicolumn{4}{|c|}{ Région géographique de résidence au moment du décès } \\
\hline Ouest & 628 & 51,3 & 1223 \\
\hline Nord & 632 & 71,7 & 881 \\
\hline Est & 175 & 40,9 & 428 \\
\hline \multicolumn{4}{|l|}{ Année du décès } \\
\hline 2011 & 293 & 60,0 & 488 \\
\hline 2012 & 294 & 58,0 & 507 \\
\hline 2013 & 418 & 55,4 & 755 \\
\hline 2014 & 430 & 55,0 & 782 \\
\hline \multicolumn{4}{|l|}{ PATHOLOGIE } \\
\hline \multicolumn{4}{|l|}{ Diagnostic de cancer le plus récent } \\
\hline Sein & 165 & 64,4 & 256 \\
\hline Tête et cou & 60 & 63,8 & 94 \\
\hline Fsophage & 38 & 57,6 & 66 \\
\hline Pancréas & 58 & 56,9 & 102 \\
\hline Hématologique & 110 & 56,4 & 195 \\
\hline Poumon et bronches & 396 & 56,5 & 701 \\
\hline Prostate & 70 & 54,5 & 124 \\
\hline Colorectal & 228 & 53,8 & 424 \\
\hline Autre & 310 & 54,4 & 570 \\
\hline
\end{tabular}

Suite à la page $28 . .$. 


\begin{tabular}{|c|c|c|c|}
\hline \multirow[t]{2}{*}{ Caractéristiques des patients (décédés) } & \multicolumn{2}{|c|}{ Plus d'un dossier de navigation } & \multirow[t]{2}{*}{ Total } \\
\hline & Nombre & $\%$ & \\
\hline \multicolumn{4}{|c|}{ Stade de la maladie au moment du diagnostic de cancer le plus récent ${ }^{\mathrm{a}}$} \\
\hline 0 & 10 & 41,7 & 24 \\
\hline I & 139 & 52,1 & 267 \\
\hline II & 190 & 61,5 & 309 \\
\hline III & 284 & 61,9 & 459 \\
\hline IV & 574 & 56,4 & 1018 \\
\hline Inconnu ${ }^{b}$ & 43 & 70,5 & 61 \\
\hline Données non disponibles ${ }^{c}$ & 112 & 48,3 & 232 \\
\hline \multicolumn{4}{|l|}{ Diagnostics antérieurs de cancer } \\
\hline Oui & 323 & 58,4 & 553 \\
\hline Non & 1112 & 56,2 & 1979 \\
\hline \multicolumn{4}{|l|}{ Décès causé par le cancer } \\
\hline Oui & 1338 & 58,1 & 2302 \\
\hline Non & 97 & 42,2 & 230 \\
\hline \multicolumn{4}{|l|}{ ISSUE DE LA MALADIE } \\
\hline \multicolumn{4}{|c|}{ Temps écoulé entre le dernier diagnostic de cancer et le décès ${ }^{d}$} \\
\hline 2 semaines ou moins & 12 & 25,0 & 48 \\
\hline 2 à 13 semaines ( 3 mois) & 134 & 36,0 & 372 \\
\hline 13 à 26 semaines (6 mois) & 154 & 53,3 & 289 \\
\hline 26 à 52 semaines ( 1 an) & 282 & 64,8 & 435 \\
\hline \multicolumn{4}{|l|}{ Lieu du décès ${ }^{e}$} \\
\hline Hôpital & 810 & 55,4 & 1461 \\
\hline Hors de l'hôpital & 332 & 56,9 & 583 \\
\hline TOTAL & 1435 & 56,7 & 2532 \\
\hline \multicolumn{4}{|c|}{$\begin{array}{l}\text { Les résultats en gras indiquent une association statistiquement significative ( } p<.05 \text { ) entre la caractéristique et le nombre de dossiers } \\
\text { mensuels de navigation (dossiers multiples). } \\
\text { a Nous avons écarté } 162 \text { patients décédés parce que leur diagnostic de cancer avait été posé avant l'adoption de la stadification concertée. } \\
\text { b Le groupement TNM du « meilleur stade » n'a pu être déterminé à cause d'un manque de critères descriptifs. } \\
\text { ' Le « meilleur stade » TNM n'a pu être déterminé à partir de la version } 2.0 \text { de la stadification concertée. } \\
\text { d Les décès ayant eu lieu après plus de } 52 \text { semaines ont été écartés, car il était impossible de vérifier si tous les patients avaient eu accès à } \\
\text { une infirmière pivot plus d'un an avant leur décès. } \\
\text { e Nous avons écarté } 488 \text { patients décédés en 2011, car le lieu du décès (hôpital ou autre) n'était pas connu pour cette année-là. }\end{array}$} \\
\hline
\end{tabular}

l'infirmière pivot avait aiguillé le patient vers une équipe de soins palliatifs (tableau 3). Seule une faible proportion des dossiers de navigation $(5,6 \%)$ où figurait un contact pour soins palliatifs mentionnait, à l'inverse, un renvoi vers l'infirmière pivot par l'équipe de soins palliatifs.

Des variations statistiquement significatives ont été observées pour certains facteurs démographiques, mais pas pour tous. Plus les patients étaient âgés, moins les dossiers de navigation mensuels étaient nombreux (tableau 1). Chez les personnes de 19 à 39 ans, 72,2\% avaient plusieurs dossiers de navigation; en revanche, la proportion était environ deux fois moindre (39,0\%) pour les personnes de 90 ans et plus. Cependant, l'association entre l'âge et la diminution des contacts pour soins palliatifs n'était pas statistiquement 
Tableau 2 : Variation des caractéristiques des patients décédés selon le dernier dossier de navigation rapportant un contact pour soins palliatifs

\begin{tabular}{|c|c|c|c|}
\hline Caractéristiques des patients décédés & \multicolumn{2}{|c|}{ Contact pour soins palliatifs } & Total \\
\hline \multicolumn{4}{|l|}{ Tranche d'âge au moment du décès } \\
\hline $19-39$ & 7 & 38,9 & 18 \\
\hline $55-69$ & 294 & 31,0 & 947 \\
\hline $70-79$ & 246 & 31,3 & 787 \\
\hline $80-89$ & 127 & 28,5 & 445 \\
\hline Femme & 354 & 29,0 & 1220 \\
\hline Homme & 422 & 32,2 & 1312 \\
\hline \multicolumn{4}{|c|}{ Région géographique de résidence au moment du décès } \\
\hline Ouest & 371 & 30,3 & 1223 \\
\hline Nord & 324 & 36,8 & 881 \\
\hline Est & 81 & 18,9 & 428 \\
\hline \multicolumn{4}{|l|}{ Année du décès } \\
\hline \multicolumn{4}{|l|}{ PATHOLOGIE } \\
\hline \multicolumn{4}{|l|}{ Diagnostic de cancer le plus récent } \\
\hline Sein & 49 & 19,1 & 256 \\
\hline Colorectal & 92 & 21,7 & 424 \\
\hline Tête et cou & 22 & 23,4 & 94 \\
\hline Hématologique & 50 & 25,6 & 195 \\
\hline Fsophage & 17 & 25,8 & 66 \\
\hline Prostate & 34 & 27,4 & 124 \\
\hline Poumon & 270 & 38,5 & 701 \\
\hline Pancréas & 43 & 42,2 & 102 \\
\hline Autre & 199 & 34,9 & 570 \\
\hline
\end{tabular}

Suite à la page $30 .$. 


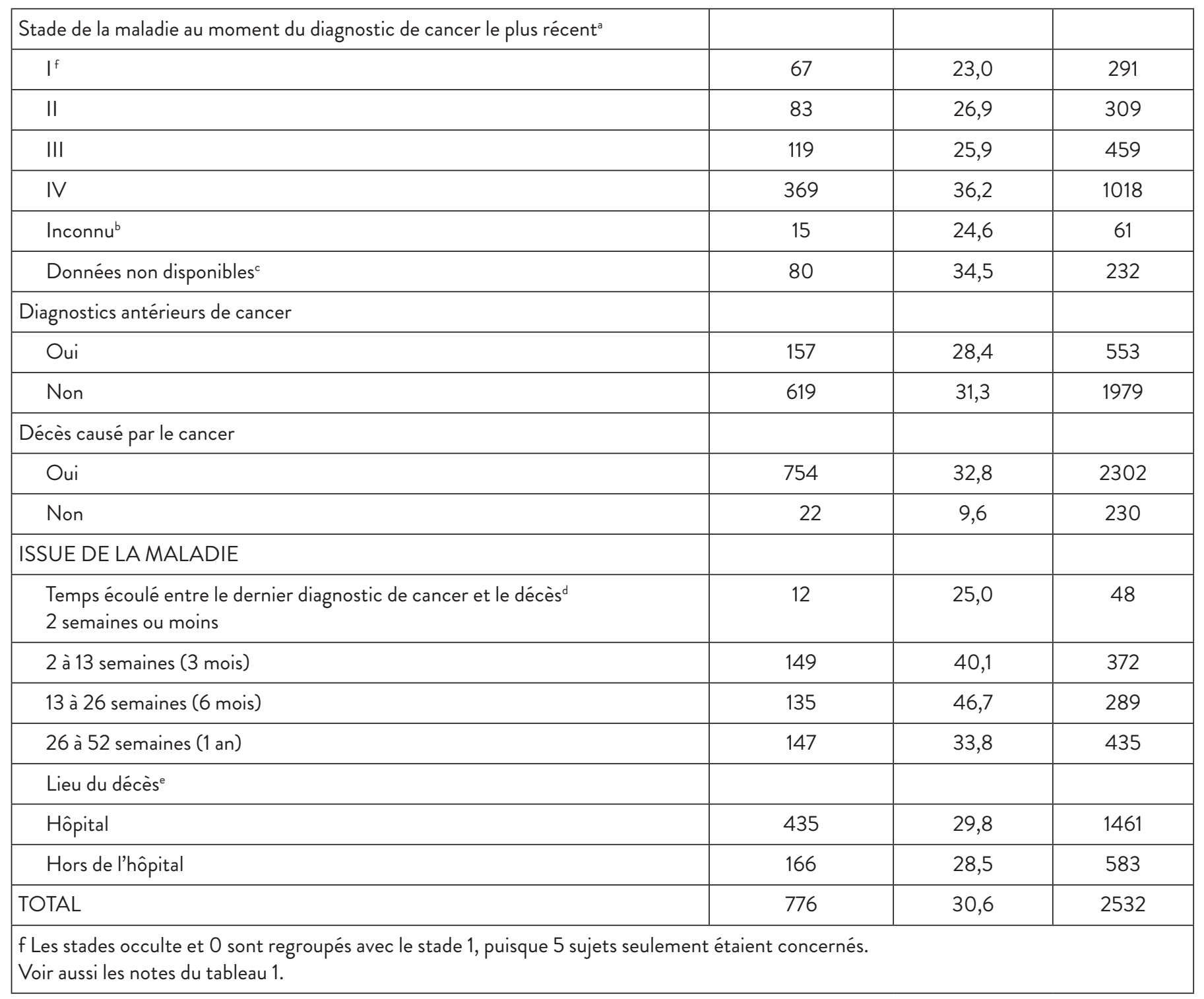

significative (tableau 2). L'association entre le sexe et la fréquence de navigation (tableau 1) ou le contact pour soins palliatifs (tableau 2) n'était pas non plus concluante. Toutefois, le lien entre la région géographique et la fréquence de navigation et de contact pour soins palliatifs était significatif. Enfin, l'année de décès était associée au contact pour soins palliatifs, mais pas avec la fréquence de navigation.

Le diagnostic de cancer était fortement corrélé au contact pour soins palliatifs, mais pas à la fréquence de navigation. Les personnes atteintes de cancer du sein avaient le taux de contact pour soins palliatifs le plus bas $(19,1 \%)$, le taux le plus élevé se trouvant chez les patients souffrant de cancer du pancréas $(42,2 \%)$ et du poumon $(38,5 \%)$. Le stade de la maladie au moment du diagnostic était significativement corrélé tant à la fréquence de navigation qu'au contact pour soins palliatifs. Bien que le taux de contact pour soins palliatifs le plus élevé ait été pour les cancers de stade IV (36,2\%), 56,4\% des patients à ce stade de la maladie présentaient aussi de multiples dossiers de navigation, une valeur très proche de la moyenne $(56,7 \%)$. Le taux de navigation multiple était toutefois plus élevé pour les stades II (61,5 \%) et III (61,9\%), la première place $(70,5 \%)$
Tableau 3 : Création d'une variable de contact pour soins palliatifs à partir du dernier dossier de navigation

\begin{tabular}{|l|c|}
\hline Base de codage du contact pour soins palliatifs & $\begin{array}{c}\text { Compte } \\
\text { (pourcentage) }\end{array}$ \\
\hline $\begin{array}{l}\text { Option unique : L'infirmière pivot aiguille le } \\
\text { patient vers les soins palliatifs }\end{array}$ & $372(47,9)$ \\
\hline $\begin{array}{l}\text { Option unique : L'équipe de soins palliatifs } \\
\text { aiguille le patient vers l'infirmière pivot }\end{array}$ & $44(5,6)$ \\
\hline Option unique : Coordination des soins palliatifs & $77(9,9)$ \\
\hline $\begin{array}{l}\text { Deux options : Aiguillage relatif aux soins } \\
\text { palliatifs, personne ayant fait la demande } \\
\text { d'aiguillage, ou coordination }\end{array}$ & $269(34,7)$ \\
\hline $\begin{array}{l}\text { Toutes : Aiguillage relatif aux soins palliatifs, } \\
\text { personne ayant fait la demande d'aiguillage, ou } \\
\text { coordination }\end{array}$ & $14(1,8)$ \\
\hline Total & $776(100)$ \\
\hline
\end{tabular}


revenant cependant au petit nombre de patients $(n=43)$ dont le stade de la maladie n'était pas connu, par manque de données dans le Système d'enregistrement des données sur le cancer pour établir la stadification.

L'existence d'un diagnostic antérieur de cancer et le lieu du décès n'étaient liés ni à la fréquence de navigation, ni au contact pour soins palliatifs. En revanche, le temps écoulé depuis le dernier diagnostic de cancer et la cause du décès étaient tous deux significativement associés à la fréquence de navigation et au contact pour soins palliatifs. Environ un tiers $(32,8 \%)$ des personnes mortes du cancer avaient eu un contact pour soins palliatifs, ce nombre diminuant à $9,6 \%$ pour les décès non attribuables au cancer.

\section{DISCUSSION}

Les résultats de la présente étude mettent en lumière les taux de navigation et de contact pour soins palliatifs des personnes en fin de vie ayant reçu un diagnostic de cancer et des services de navigation. Parmi les patients de l'étude décédés des suites du cancer après avoir reçu des services de navigation, 32,8 \% avaient eu un contact pour soins palliatifs avec l'infirmière pivot. Étant donné que seulement $40 \%$ des patients emportés par le cancer avaient reçu des services de navigation (Park, Johnston, Urquhart, Walsh et McCallum, 2018), cela porte à $13,1 \%$ seulement $(32,8 \%$ multiplié par 0,4$)$ la proportion de patients décédés des suites du cancer ayant eu un contact pour soins palliatifs avec l'infirmière pivot. Ce taux est bas comparativement à la proportion de patients cancéreux bénéficiant de programmes de soins palliatifs rapportée par d'autres auteurs. Par exemple, Gao, Johnston, Lavergne et McIntyre (2011) mentionnent un taux de $72 \%$ et Craigs, West, Hurlow, Bennett et Ziegler (2018), un taux de 65 \%. Dans chacune de ces études, le lien entre la variation des taux et les facteurs découlant de la démographie, de la pathologie et de l'issue de la maladie était statistiquement significatif.

Dans notre étude, les patients plus âgés avaient souvent un seul dossier de navigation, ce qui veut dire que les infirmières pivots intervenaient sans doute moins souvent et pendant moins longtemps auprès de cette clientèle. En outre, les patients plus âgés étaient moins susceptibles de recevoir des services de navigation (Park, Johnston, Urquhart, Walsh et McCallum, 2018), ce qui les rendrait doublement vulnérables : en plus de recevoir moins de services de navigation, ils n'en bénéficieraient souvent que pour une courte période seulement (moins d'un mois). Duthie, Strohschein et Loiselle (2017) rapportent qu'avec l'âge, les patients ayant reçu un diagnostic de cancer risquent davantage de souffrir de comorbidités multiples, ce qui ajoute à la complexité de leurs soins et influence, en retour, les possibilités de traitement, le pronostic et les besoins en matière de soins. Par conséquent, les personnes plus âgées aux prises avec le cancer ne reçoivent peut-être pas les services de navigation dont elles ont besoin; il est également possible que leurs besoins en cette matière soient satisfaits par d'autres professionnels de la santé. À l'opposé, les personnes plus jeunes reçoivent peutêtre plus de services de navigation qu'elles n'en ont besoin ou alors elles ne trouvent pas satisfaction à leurs besoins auprès des autres membres de leur équipe de soins.
Le diagnostic de cancer influençait considérablement le fait de recevoir ou non des services de navigation (Park, Johnston, Urquhart, Walsh et McCallum, 2018). Toutefois, selon notre étude, ni la fréquence de navigation (tableau 1), ni le taux de contact pour soins palliatifs (tableau 2) ne variaient selon le diagnostic de cancer une fois que le patient avait consulté l'infirmière pivot. Plus précisément, 30,6 \% des sujets décédés avaient eu un contact pour soins palliatifs, mais ce taux variait entre 20 et $40 \%$ selon le site du cancer et le stade de la maladie au moment du diagnostic (tableau 2). Les variations du taux $\mathrm{du}$ contact pour soins palliatifs s'expliquent par une combinaison de deux facteurs, soit le moment dans la trajectoire du cancer où les services de navigation étaient offerts et la durée de survie.

C'est le cancer du sein qui présentait le taux le plus faible $(19,1 \%)$ de contact pour soins palliatifs. Bien que les patientes atteintes de cancer du sein aient un taux de navigation plus élevé que la moyenne (Park, Johnston, Urquhart, Walsh et McCallum, 2018), les résultats de notre étude s'accordent à ceux des études antérieures sur le dépistage, le diagnostic précoce et le traitement rapide du cancer du sein (Freeman, 2006; Mertz et al., 2017). Les patientes atteintes de cancer du sein ont une période de survie relativement longue (SCC, 2017); ainsi, la navigation peut avoir eu lieu des années avant le décès, à un moment où les soins palliatifs ne constituent pas une priorité.

Dans notre étude, les patients souffrant d'un cancer de la prostate avaient un taux de contact pour soins palliatifs dans la moyenne $(27,4 \%)$. Ce taux était plus élevé que pour les cas de cancer du sein $(19,1 \%)$ malgré le fait que ces patients survivent plus longtemps que les femmes touchées par le cancer du sein (SCC, 2017). Nos résultats s'expliquent peut-être par le fait que les patients atteints de cancer de la prostate avaient le taux le plus bas de navigation, une situation qui découle probablement de la tendance souvent préconisée au moment du diagnostic de ce type de cancer d'attendre et d'observer l'évolution de la maladie, ainsi qu'au fait que les urologues ne font pas partie du programme d'oncologie (Park, Johnston, Urquhart, Walsh et McCallum, 2017). Par conséquent, lorsque les patients atteints de cancer de la prostate voient une infirmière pivot, leur maladie est possiblement très avancée. Il est donc possible que les soins palliatifs soient alors plus pertinents pour eux que pour les personnes avec un cancer du sein ayant reçu des services de navigation.

C'est le cancer du pancréas qui présentait le taux le plus élevé de contact pour soins palliatifs (42,3\%). Le taux de navigation, lui, était inférieur à la moyenne (Park, Johnston, Urquhart, Walsh et McCallum, 2017), vraisemblablement parce qu'une forte proportion des patients n'avaient pas été vus en oncologie (Hurton et al., 2017). Étant donné leur temps de survie relativement court (SCC, 2017), il n'est pas surprenant qu'au moment où ils ont consulté une infirmière pivot, il y ait eu contact pour soins palliatifs dans environ la moitié des cas.

Les liens avec le stade de la maladie ne sont pas surprenants vu le rôle facilitant de l'infirmière pivot dans le traitement du cancer. Aux stades II et III, le nombre de dossiers de navigation multiples était plus élevé que la moyenne; il était cependant plus faible lorsque le cancer n'en était qu'à un stade 
précoce. Or, les stades II et III exigent généralement des traitements plus longs et plus agressifs. Bien que le taux de dossiers mensuels multiples soit plus élevé chez les patients dont le stade de cancer était inconnu au moment du diagnostic, ces derniers étaient, en amont, les moins susceptibles d'être aiguillés vers une infirmière pivot (Park, Johnston, Urquhart, Walsh et McCallum, 2018). Plus la maladie était avancée, plus il était probable que la dernière séance de navigation ait été consacrée à un contact pour soins palliatifs. Ce n'est pas surprenant, puisque la probabilité de succomber au cancer, et donc d'avoir besoin de soins palliatifs, augmente avec le stade au moment du diagnostic.

Lorsque le diagnostic avait été posé dans les deux semaines précédant le décès, le taux de contact pour soins palliatifs n'était pas le plus élevé $(25,0 \%)$. C'est plutôt lorsque le diagnostic intervenait durant la période de trois à six mois précédant le décès que le taux était à son maximum (46,7 \%) (tableau 2). Cette tendance est conforme à celles d'autres études, qui ont montré que, lorsque le décès survenait rapidement après le diagnostic, les patients étaient moins susceptibles d'être aiguillés vers un programme de soins palliatifs (Gao, Johnston, Lavergne et McIntyre, 2011). Toutefois, notre étude a révélé que les infirmières pivots voyaient bel et bien les patients qui décédaient dans les trois mois suivant un diagnostic de cancer; il serait donc avisé d'accroître les contacts pour soins palliatifs de la part des infirmières pivots pour les patients recevant un pronostic de trois mois ou moins.

S'il est vrai que les infirmières pivots facilitent les soins palliatifs pour environ un tiers $(33,8 \%)$ des patients adultes qui finissent par mourir des suites du cancer, les soins palliatifs sont aussi dénotés pour environ un patient sur dix (9,6\%) qui décède pour une autre raison (tableau 2). Jusqu'à présent, peu de recherches ont été publiées sur le rôle des infirmières pivots dans l'accès aux soins palliatifs des survivants du cancer dont la fin de vie est précipitée par d'autres maladies.

\section{Forces, limites et prochaines étapes}

En publiant les premières études épidémiologiques en population sur la navigation des patients cancéreux en fin de vie, nous confirmons la pertinence de notre contribution pour comprendre la navigation vers les soins palliatifs. Nos travaux montrent qu'il est possible non seulement de faire une étude en population sur la navigation, mais aussi de mettre l'accent sur l'accès aux soins palliatifs. Bien que la Nouvelle-Écosse dispose de données basées sur la population pour suivre les tendances au fil du temps, il faut tout de même établir des mesures démographiques comparables afin de pouvoir les mettre en parallèle avec les données des différentes provinces.

D'autres recherches seront nécessaires pour expliquer les résultats d'iniquités ou de possibles lacunes dans les services que pourrait venir combler la navigation des patients atteints de cancer et qui, sans elle, ne seraient pas disponibles. Par exemple, dans la présente étude, la région géographique influençait le nombre de séances de navigation; quant aux contacts pour soins palliatifs, ils variaient en fonction de l'année et de la zone géographique. Or, l'est de la province (où le taux de contact pour soins palliatifs est le plus faible, c.-à-d.de
$18,9 \%)$ dispose d'un programme de soins palliatifs bien établi intégré à son programme d'oncologie; les infirmières pivots n'avaient donc pas autant à participer aux soins palliatifs. En revanche, une partie du nord de la province doit composer avec une pénurie de spécialistes en soins palliatifs, et c'est justement dans cette région que le taux de contact pour soins palliatifs était le plus élevé (36,8\%).

Données en main, nous pouvons maintenant formuler des hypothèses. Les taux sont-ils indicateurs de besoins? Comment s'expliquent les variations de taux? Le nombre de contacts pour soins palliatifs générés par l'infirmière pivot est-il adéquat pour les personnes âgées atteintes de cancer? Pourquoi les patients ayant vécu moins de trois mois après le diagnostic de cancer présentaient-ils des taux de contact pour soins palliatifs plus faibles que ceux qui avaient survécu plus longtemps (c.-à-d. trois à six mois) après leur diagnostic?

Les variables relatives à la navigation pour soins palliatifs extrapolées à partir de nos données sur la population sont limitées. La formulation de recommandations visant des résultats particuliers pourrait orienter l'expansion des suivis des soins palliatifs et terminaux effectués par les infirmières pivots (Hauser et al., 2011; Fiscella et al., 2011; Watson, Vimy, Anderson, Champ et DeIure, 2016). Il est également conseillé de vérifier la qualité des données et leur interprétation, par exemple dans le cas des personnes décédées d'une cause non liée au cancer et qui étaient vues par une infirmière pivot pour une démarche palliative. Il conviendrait aussi d'intégrer certaines corrections aux calculs afin de confirmer les associations entre les variables. Dans l'avenir, le suivi des populations pourrait permettre d'évaluer le rôle de la navigation pour améliorer les résultats d'intérêt (Paskett, Harrop et Wells, 2011).

\section{CONCLUSION}

Bien que d'autres études en population sur l'accès aux soins palliatifs aient déjà été publiées, le présent article est le premier à aborder la fréquence de contact pour soins palliatifs des patients cancéreux en fin de vie qui ont eu accès à des services de navigation. Notre étude souligne l'importance d'évaluer la fréquence de navigation et de contacts pour soins palliatifs des patients atteints de cancer qui entrent en phase terminale. Nos études en population sur la navigation des patients cancéreux en fin de vie constituent le point de départ d'un examen plus approfondi du rôle que jouent les infirmières pivots en oncologie pour faciliter l'accès aux soins palliatifs des personnes atteintes de cancer. Le suivi des populations permet donc de brosser un portrait des personnes qui reçoivent des services de navigation, ainsi que de la durée et du motif de l'offre de services.

\section{FINANCEMENT}

Grace Park a reçu la bourse d'études Chase Summer de la Dalhousie Medical Research Foundation. La Régie de la santé de la Nouvelle-Écosse, par l'entremise de son programme de soins du cancer, a aussi apporté un appui non financier. 


\section{RÉFÉRENCES}

Comité directeur de la Société canadienne du cancer (2010). Statistiques canadiennes sur le cancer 2010. Toronto, ON : Société canadienne du cancer. [en ligne] http://www.cancer.ca/ / media/cancer.ca/CW/cancer\%20information/cancer\%20101/ Canadian\%20cancer\%20statistics/Canadian-Cancer-Statistics2010-FR.pdf?la=fr-CA

Comité directeur de la Société canadienne du cancer (2017). Statistiques canadiennes sur le cancer 2017. Toronto, ON : Société canadienne $\mathrm{du}$ cancer. [en ligne] http://www.cancer.ca/ / media/cancer.ca/CW/cancer\%20information/cancer\%20101/ Canadian\%20cancer\%20statistics/Canadian-Cancer-Statistics2017-FR.pdf?la=fr-CA

Craigs, C.L., West, R.L., Hurlow, A., Bennett, M.I., \& Ziegler L.E. (2018) Access to hospital and community palliative care for patients with advanced cancer: A longitudinal population analysis. PLOS ONE 13:8, e0200071.

Duthie, K., Strohschein, F.J., \& Loiselle, C.G. (2017). Living with cancer and other chronic conditions: Patients' perceptions of their healthcare experience. Canadian Oncology Nursing Journal, 27(1), 43-48.

Fillion, L., Cook, S., Veillette, A.M., deSerres, M., Aubin, M., Rainville, F., ... Doll, R. (2012). Professional navigation: A comparative study of two Canadian models. Canadian Oncology Nursing Journal, 22(4), 257-277.

Fiscella, K., Ransom, S., Jean-Pierre, P., Cella D., Stein, K., Bauer, J.E., ... Walsh, K. (2011). Patient-reported outcome measures suitable to assessment of patient navigation. Cancer, 117(15 Suppl.), 3603-3617.

Fitch, M. (2017). Focusing on care of older adults with cancer. Canadian Oncology Nursing Journal, 27(3), 208.

Freeman, H. (2006). Patient navigation: A community based strategy to reduce cancer disparities. Journal of Urban Health, 83(2), 139-141.

Freeman, H.P., \& Rodriguez, R.L. (2011). History and principles of patient navigation. Cancer, 117(15 Suppl.), 3539-3542.
Gao, J., Johnston, G.M., Lavergne, M.R., \& McIntyre, P. (2011). Identifying population groups with low palliative care program enrolment using classification and regression tree analysis. Journal of Palliative Care, 27(2), 98-106.

Hauser, J., Sileo, M., Araneta, N., Kirk, R. Martinez, J., Finn, K., ... Rodrigue M.K. (2011). Navigation and palliative care. Cancer, 117(15 Suppl.), 3585-3591.

Hurton, S., Urquhart, R., Kendell, C., Jorgensen, M., Porter, G., Levy, A., \& Moilinary, M. (2017). Variations in medical oncology utilization for pancreatic cancer patients in Nova Scotia. Journal of the Pancreas, 18(10), 62-68.

Lavergne, M.R., Johnston, G.M., Gao, J., Dummer, T.J.B., \& Rheaume, D.E. (2011). Variation in the use of palliative radiotherapy at end of life: Examining demographic, clinical, health service, and geographic factors in a population-based study. Palliative Medicine, 25(2), 101-110.

Mertz, B.G., Dunn-Henriksen, A.K., Kroman, N., Johansen, C., Andersen, K.G., Andersson, M., ... Envold Bidstrup, P. (2017). The effects of individually tailored nurse navigation for patients with newly diagnosed breast cancer: A randomized pilot study. Acta Oncologica, 56(12), 1682-1689.

Park, G., Johnston, G., Urquhart, R., Walsh G., \& McCallum, M. (2018). Comparing enrolees to non-enrolees of cancer patient navigation at end of life. Current Oncology, 25(3),e184-e192.

Paskett, E.D., Harrop, J.P., \& Wells, K.J. (2011). Patient navigation: An update on the state of the science. CA Cancer Journal for Clinicians, 61(4), 237-249.

SAS software, version 9.4. Copyright (C) 2002-2012 SAS Institute Inc. Cary, NC, USA.

Walkinshaw, E. (2011). Patient navigators becoming the norm in Canada. Canadian Medical Association Journal, 183(15), e1109-e1110.

Watson, L.C., Vimy, K., Anderson, J., Champ, S., \& DeIure, A. (2016). Developing a provincial cancer patient navigation program utilizing a quality improvement approach Part three: Evaluation and outcomes. Canadian Oncology Nursing Journal, 26(4), 276-284. 